\title{
Breathing Together: Children Co-constructing Asthma Self-Management in the United States
}

\author{
Julie Spray $^{1,2}$ D $\cdot$ Jean Hunleth ${ }^{1}$
}

Accepted: 24 December 2021

(C) The Author(s), under exclusive licence to Springer Science+Business Media, LLC, part of Springer Nature 2022

\begin{abstract}
Pediatric asthma management in the U.S. is primarily oriented around caregivers. As evident in policy, clinical literature and provider practices, this caregiver-centric approach assumes unidirectional transfer of practices and knowledge within particular relational configurations of physicians, caregivers, and children. Reflecting broader societal values and hierarchies, children are positioned as passive recipients of care, as apprentices for future citizenship, and as the responsibility of parents who will train them in the knowledge and labor of asthma management. These ideas, though sometimes contradictory, contribute to a systemic marginalization of children as participants in their health care, leaving a conceptual gap regarding children's inclusion in chronic illness management: what children's roles in their health care are or should be. We address this conceptual gap by asking, what does pediatric asthma management look like when we center children, rather than caregivers in our lens? We draw data from a study of asthma management in St. Louis, Missouri, and Gainesville, Florida, which included 41 caregivers, 24 children, and 12 health-care providers. By asking children to show us how they manage asthma, we find that children actively co-construct health practices within broader interdependencies of care and the structural constraints of childhoods.
\end{abstract}

Julie Spray

j.spray@auckland.ac.nz

1 Division of Public Health Sciences, Washington University School of Medicine, St. Louis, MO, USA

2 School of Population Health, Section of Social and Community Health, University of Auckland, Auckland, New Zealand 
Keywords Asthma · Self-management · Childhood · Responsibility ·

Care

\section{Introduction}

Since the neoliberal turn in health care of the 1990s, the dominant approach to asthma treatment in the United States has been "self-management." The selfmanaging patient, theoretically under physician guidance, holds responsibility for monitoring symptoms and administering and adjusting pharmaceutical regimes (Aroni et al. 2003). As a policy, however, self-management was designed with the adult patient in mind, leaving caregivers or health-care providers to interpret the roles of the approximately six million children with asthma in the United States.

Indeed, children only appear as shadows in the "apparatus" of asthma management: the policies, guidelines, and clinical models that scaffold the roles and responsibilities that patients, caregivers, and clinical providers respectively hold in chronic illness care. As we will show, self-management policy typically assumes caregivers - often mothers - as proxy patients, and asthma guidelines position children as passive recipients of care or "problems." ${ }^{1}$ Clinical practices based on developmental models can be absent any framework for children's participation before adolescence. This uncertainty surrounding children's roles in their health care has left, by default or elision, a clinical approach to pediatric asthma management that is unreflexively oriented around caregivers. Children, if considered at all, are often positioned as apprentices who are training for future adulthood by receiving education and gradually learning to 'take responsibility' for their asthma management under parental supervision.

Children's ambiguous, and sometimes contradictory positioning in their asthma management, whether as irrelevant, caregivers' responsibilities, or 'apprentices,' therefore signals a large conceptual gap in self-management policy: what are, or should be, children's roles in chronic illness management? In this article, we begin to address the question of what children's roles might be by first asking about what roles children are taking. How are children developing and practicing asthma management, what roles are they taking and when, and how do they see themselves as self-managing or managed patients? The confusion about who children are that necessitates our questions is understandable given the broader societal values and hierarchies that influence constructions of childhood in public health. Scholars have noted children's invisibilization in society (James, Jenks, and Prout 1998) and in medical fields (Bluebond-Langner 1978; Ford et al. 2018; Gabe, Olumide, and Bury 2004; Hunleth 2017; Sweis 2021), where cultural notions of children as incompetent, vulnerable, innocent, and people who ought not be responsible are conflated with assumptions that children's activities are inconsequential (Spray 2020). The "apprentice" framing echoes the developmental models that underpin pediatric medicine (Woodhead 2009) with a neoliberal twist, positioning parents as

\footnotetext{
${ }^{1}$ Caregivers are also seen as the problem in this way of thinking. As proxy patients, they are the ones who are labeled nonadherent and held responsible for any child behavior or worsened clinical outcomes.
} 
agents 'responsible for responsibilizing' their children toward a future as citizens who take on the labor and accountability for their illness management. As bearers of the asthmatic bodies, children's efforts are critical to their health, yet the idea that children do critical care work is fraught for caregivers, researchers, providers and policymakers grappling with morally and culturally contested notions of what children's roles and responsibilities ought to be. With ideology concealing children's realities, self-management policy is left with a shadow version of childhood resembling a sole caregiver (usually a mother) unidirectionally passing down tasks and responsibilities to an agendaless child whose only constraints are those enacted or mediated by the caregiver.

The past four decades of childhood studies research, however, strongly contraindicates this passive version of the child patient. An established body of literature has demonstrated that children are social actors with agency, who construct their own interpretations of illness and responsibility, solicit or resist care, seek control over their bodies, and generate their own health-care practices in collaboration with other actors (Bluebond-Langner 1978; Christensen 1999; Clark 2003; James 1993; Mayall 1993; Prout 1986; Prout, Hayes, and Gelder 1999; Spray 2020; Sweis 2021). This literature would suggest that, while caregivers likely influence children's self-management, children generate, rather than merely acquire health practices. A focus on children's agency represents an important corrective to the assumptions of the passive incompetent child embedded in the apparatus of much pediatric illness management. Yet, as recent scholars have argued, an emphasis on children's agency risks under-analysis of the ways in which children's actions are constrained by social structures or material scarcity (Bluebond-Langner and Korbin 2007; Allerton 2016). As Hunleth (2019:157) cautions, "children are, in fact, agents, but they are not "super agents," we are wise to remember. None of us are."

While agency is, thus, too blunt an instrument, so too is constraint. To be sure, children who are managing illness do encounter constraint, including, for example, stark material disadvantage that renders health-care unaffordable, policy that limits access to medications, and laws governing children's independent mobility and decision-making. If many caregivers are, as we have found (Hunleth et al. 2020), limited in their ability to move out of unhealthy housing conditions, to prevent housemates from smoking, or to access transport to medical care, then children are even further constrained from taking such actions. Yet, as a broad literature in medical anthropology has established, illness involves not only biological but social processes; much of children's asthma management occurs through everyday, relational interactions. Such interactions are not only there to enable or constrain children's practices, but sites for constituting and enacting who children and others are to one another. The language of agency and constraint is therefore insufficient to represent how, for example, a child may decline to "take responsibility" for his asthma management because he recognizes his mother's need to enact care, or how two brothers might turn asthma management from stoic competition to mutual protection. What is needed, then, is an approach to children's roles in medicine that encompasses the complex ways that children engage with resources-including 
caregivers - and negotiate their structural and relational positions in families and wider society.

In approaching the question of who children are in asthma management, therefore, we locate children within ecologies of social relations, interdependencies, resources, institutional structures and systems, all of which shape their health practices. Through ethnographic and online visits in Gainesville, Florida, and St. Louis, Missouri, we invited 24 children with asthma or siblings to tell us how they participate in their asthma care and further contextualized children's perspectives through interviews with 41 caregivers and 12 asthma care providers. We emphasize children's situatedness as children, meaning their childhood status structures the people available to children, how children can and wish to relate to those others, and the environments and institutions children work within. By shifting the center of our lens to children, rather than caregivers, we reconstitute the shadow child of selfmanagement policy as a social actor who co-constructs, with a variety of other actors, an illness management that is enfolded within interdependencies, mutual responsibility and obligation, which are in turn shaped by cultural, socio-economic, and racialized realities of domestic life. In doing so, we also map a way of analyzing children's practices with views of both agency and constraint and that which falls in between. And, we hope to offer a view of children's care practices that may advance clinical models of pediatric chronic illness management.

\section{Children's Invisible Roles and Responsibilities in Pediatric Asthma Self- Management}

Though originally envisioned as a collaborative partnership between patient and physician, since the 1990s self-management approaches have functioned to shift the responsibility and labor for health care from the State onto the patient by centering self-surveillance and adherence to pharmaceuticals (Trnka 2017). Clinical versions of asthma self-management typically focus on patient self-administration of daily inhaled corticosteroids (ICS) to control inflammation and prevent symptoms, alongside short-acting inhaled beta-agonist (SABA) bronchodilators ("rescue" inhalers) to relieve acute symptoms. In practice, asthma self-management involves complex work and specialized knowledge to detect symptoms, adjust dosages, and incorporate the mental labor of pharmaceutical management and trigger avoidance into the multiple temporal and spatial dimensions of daily life (Mammen et al. 2018; Spray et al. 2021; Hunleth et al. 2020).

As the inhabitants of the asthmatic body, children must and do play critical roles in their asthma management. Children must learn to recognize their symptoms and decide which of many possible actions to take (notifying adults, self-administering medication, trying alternative techniques). Children may also make decisions regarding trigger avoidance (for example, moderating their physical activity). A number of studies have shown that children are tasked with administering their own medication from young ages (Orrell-Valente et al. 2008; Meah et al. 2010; McQuaid et al. 2003; Winkelstein et al. 2000), responsibilities which involve substantial mental labor, memory work and decision-making skills. Multiple studies have 
demonstrated that children hold unique, and often, more accurate knowledge of their asthma than their caregiver, including their symptom frequency, use of controller medications and non-prescribed self-management strategies such as limiting activity (Davis et al. 2011; Petsios et al. 2011; Guyatt et al. 1997).

Children are therefore highly implicated in their own asthma management from a young age. Yet the story of children's asthma management in the United States has been one that dismisses children and centers adult caregivers. Though some of the earliest uses of the term "self-management" are in relation to pediatric chronic illness (e.g., Creer 1976), exactly who is doing the self-managing is frequently glossed over in an adult-centric health literature based in presumptions of a standard adult patient. The majority of pediatric self-management studies focus on education and training for children in self-management behaviors and do not grapple with the unique challenges and issues associated with applying to children a policy approach designed for adult patients (Modi et al. 2012). Meanwhile, U.S. policy explicitly locates responsibility for children with their parents as a corollary of parental rights (Guggenheim 2005), and tends to be founded on particular, uncritical conceptions of childhood: that children are passive recipients of parental care; that children's lack of competency precludes accountability for health management; and that any delegation of care tasks is the parent's responsibility. These assumptions implicitly transfer self-management-designed with autonomous adult patients in mind-onto parents or guardians as patient proxies, with vague expectations that children will 'transition' at some point into independent self-management.

These assumptions that children's roles are irrelevant or only parental concerns are reflected in the guidelines, clinical literatures, and health-care services that scaffold pediatric asthma management. First, children are largely invisible in asthma guidelines, positioned as passive recipients of care and education until adolescence. For example, in the 201-page Global Initiative for Asthma (GINA) guidelines the only acknowledgement that children may participate in their health care reads: "young children can be taught simple asthma management skills" (GINA 2010:72). Similarly, the 440-page U.S. asthma guidelines (NHLBI 2007) briefly mentions that older children and adolescents should be involved in establishing goals for therapy (297), while framing these children as problems who may "fail to recognize the danger of poorly controlled asthma" or "may not accept having a chronic illness." 2 The guidelines do little to acknowledge the value of children's contributions to their care, nor suggest any indicators of when or how children should be involved in their self-management, and offer no guidance on supporting the negotiation of care responsibilities within families.

Second, only a sparse literature considers children's roles in their selfmanagement. Typically framing children's participation in terms of dyadic "transfer of responsibility," this literature addresses questions such as to the amount of

\footnotetext{
${ }^{2}$ A 2020 report presents focused updates to the 2007 guidelines. The updates primarily focus on therapeutic recommendations, and there are no additions to self-management or communication guidance. Interviews and focus groups with providers, adult patients, and caregivers of child patients contributed to the recommendations, but no children were included (NHLBI 2020).
} 
responsibility children are, can, or should be holding. ${ }^{3}$ Like pediatric medicine more broadly in the U.S., this literature often relies on developmental models to indicate when children are allocated which responsibilities (Brown et al. 2010; Buford 2004; Yoos 1994; Pradel et al. 2001), and in the absence of alternative markers, usually reverts to age as a proxy for developmental stage. For example, the "leadership" model of child asthma management positions caregivers as "transferring" roles to children in a stepwise fashion, from "provider" through to "CEO" (Kieckhefer and Trahms 2000). Such conceptualizations are based on developmental models that view children as kind of "apprentices" for adulthood, assuming a unidirectional transfer of practices, knowledge and responsibility within particular relational configurations of physicians, caregivers, and children, while black-boxing how actual processes of self-management are distributed.

In contrast with apprentice models, childhood studies views children as complete and agentive social actors who socialize themselves and each other (James 2013). A growing literature in the social science of child health shows that children are not passive recipients of care and training, but actively participate in their health through "unseen and underestimated practices" of interpreting experience, coconstructing meaning, managing bodies, and navigating the world through their structural position as children (Spray 2020:193). Importantly, this research demonstrates that overlooking children has consequences: for child and family health, relationships, and the success of health management strategies (Hunleth 2017; Spray 2020). Adult assumptions or moral judgements about children's care work or other forms of labor can obscure children's perspectives and the reality that in many circumstances, children's work is valuable, necessary, and important to their wellbeing (Reynolds 1991; Hunleth 2017; Nieuwenhuys 1996; García-Sánchez 2018). When children are positioned as apprentices in training for adulthood, their contributions are devalued as 'training' while the various ways they 'take responsibility'-for self-care, for siblings, for family finances-are ignored, minimized as 'learning,' or condemned as irresponsible parenting. Moreover, a view of asthma management that posits the clinician or [white, middle class] parental conception of what asthma management entails as the standard toward which children are being 'trained' risks missing the range of ways children engage in asthma management beyond that which is physician prescribed or caregiverdirected. Treating children as apprentice adults means ignoring how they must negotiate their particular ecological position and the constellation of social and material resources, relations, and constraints that structure their self-management practices. Children do not and cannot manage their illnesses as adults do or, necessarily, as adults may wish them to. Moreover, in a politico-cultural milieu that expects parents_-namely mothers_- to be all knowing and personally responsible for their children's health, overlooking children's agency can mean additional burdens

\footnotetext{
3 Examples include: Buford 2004; Brown et al. 2010; Ekim and Ocakci 2013; Kieckhefer and Trahms 2000; Kieckhefer et al. 2009; McQuaid et al. 2003; Munzenberger, Secord, and Thomas 2010; Newbould, Smith, and Francis 2008; Orrell-Valente et al. 2008; Walders, Drotar, and Kercsmar 2000. This literature variably encourages caregiver handover of responsibilities for asthma management to children and attributes children's non-adherence to caregivers handing over too much responsibility (e.g. Walders, Drotar, and Kercsmar 2000; Wade et al. 1999).
} 
and blame for caregivers who cannot be everywhere and do everything (Hunleth 2017). Attending to the reality of children's engagement in their health care is therefore critical to an effective, ethical, and equitable public health.

\section{Participants and Methods}

Our understandings of children's participation in their asthma management are based on a large, multi-sited, interdisciplinary study of asthma caregiver risk perception (Table 1). This research is situated in two U.S. sites: St. Louis, Missouri, and Gainesville, Florida. Both cities are marked by racial segregation and economic inequalities that locate Black families of low socio-economic status in distinct geographical areas characterized by inadequate access to basic needs, public transportation, and health-care facilities. These inequities translate into stark health disparities, including for asthma; emergency department visits have been reported to be 8.5 and 6 times higher for Black than white residents of St. Louis and Alachua county respectively (City of St. Louis Department of Health 2018; Florida Department of Health n.d.) and pediatric asthma "hotspots" have been identified in the predominantly Black and low-income communities of North St. Louis and East Gainesville (Harris 2019).

We used a range of data collection methods with caregivers, children, and clinicians, to elicit perspectives from the multiple people involved in asthma management. As the first stage of the larger study, one of us $(\mathrm{JH})$ led interviews with 41 caregivers of a child with asthma (16 Gainesville; 25 St. Louis), recruited through university or community research networks, and flyers placed in doctors' offices and community spaces. The interviews were based on a narrative interview guide that aimed to elicit the social contexts, perceptions and experiences of caring for a child with asthma over the life course. We describe the methodology in detail in Hunleth and Spray et al. (2020).

Identifying the limitations of caregiver-only perspectives, we followed up these interviews with a child and household ethnographic project approximately one year after initiating the caregiver interviews. Using a small team of researchers, we aimed to visit families at home to gain children's perspectives on asthma through child-focused activities and ethnographic observation (Spray, Fechtel, and Hunleth in press). We took a child-centered approach, which views children as social actors who hold unique insight into their own experiences and who are capable of meaningful participation in both their own care and in research (Christensen and James 2000). We modeled our approach on our prior household-based work with children (Hunleth 2011; Spray 2020) and on the work of Cindy Dell Clark (2003) who studied children's coping with asthma and diabetes through a range of childcentered activities (play, drawing, photography) that she explored with children across two home visits.

Our original intention, to visit 12 families in their homes twice within a threemonth period, was thwarted by Covid-19. We were able to recruit and make the first visit to the homes of 8 original caregivers, and one additional family, to work with 14 children with asthma and 5 siblings before it became unsafe to make further 


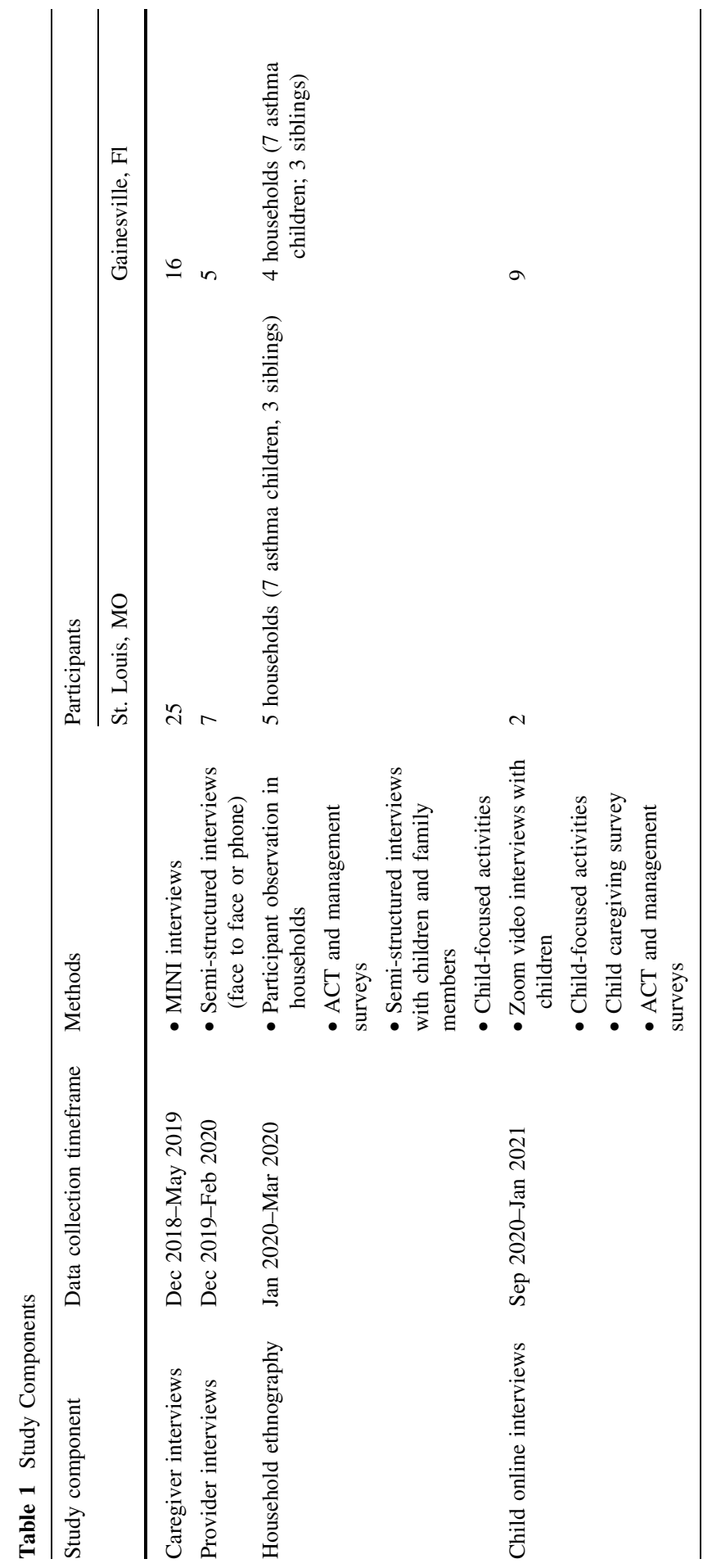


Table 2 Participant characteristics

\begin{tabular}{lc}
\hline Caregivers $(N=41)$ & $N(\%)$ \\
\hline Gender & \\
Female & $34(83)$ \\
Male & $7(17)$ \\
Racial background ${ }^{a}$ & \\
Black/African American & $30(67)$ \\
White & $11(24)$ \\
Asian & $1(2)$ \\
American Indian/Alaska Native & $3(7)$
\end{tabular}

Economic status ${ }^{\mathrm{b}}$

Low

Not-low

Insurance

Private-Employer paid

Private-marketplace

Medicaid

No insurance

Did not state

Education

Graduate, professional, or bachelor's degree

Associate's degree or vocational/technical school

High school degree or equivalent

Less than high school

Child Participants $(N=24)$

Age

$<6$

$6-9$

$10-12$

13-16

Gender

$\mathrm{M}$

$\mathrm{F}$

Racial background ${ }^{a}$

Black/African American 17

$\begin{array}{ll}\text { White } & 7\end{array}$

American Indian/Alaska Native 4

${ }^{\text {a Some participants identified with more than one category. One caregiver (2\%) also identified as Hispanic }}$ ethnicity

${ }^{\mathrm{b}}$ Based on reported financial status and/or ability to pay an unexpected $\$ 500$ medical bill not covered by insurance 
visits. Six months later, we resumed the child-focused activities online, and held follow-up interviews with 6 original children and 5 newly recruited children. In total, we worked with 19 children with asthma aged 6-16, and 5 siblings aged 4-8, across the various modalities (Table 2). Most children were African American (71\%) and from low socio-economic status households (92\%). During the household visits and online visits, we administered an asthma management survey and standard validated caregiver-proxy (duRivage et al. 2017) and child asthma control tests (Liu et al. 2007) to evaluate symptoms and pharmaceutical usage. Children represented a wide spectrum of asthma severity and indicated both under- and over-diagnosis. In general, children's asthma appeared to be undertreated, and only five of the children we spoke to were on controller medications (with varying degrees of adherence).

For both the household visits and online interviews, we followed a mosaic approach (Clark and Moss 2011) to tailor the research methods to children's needs (e.g., age, interest, number of children involved in the visit) by designing a menu of activities from which children could choose [Table 3]. These activities included drawing, sensory materials, interviews, and creating a 'welcome pamphlet' with advice for a child who has just learned they have asthma. The activities were designed to elicit children's experiences with and conceptions of asthma, and their asthma management practices, and sustain children's engagement during in-person or online interactions. Younger children tended to engage more with drawing and sensory materials, and children's representations could helpfully illustrate embodied and relational aspects of asthma experience (for example by showing experiential aspects of breathing difficulties using balloons, pipe cleaners and Play Doh) (Spray, Fechtel, and Hunleth in press). Older children could prefer talking about their experiences, though the drawing materials could also facilitate rapport as researchers also drew together with participants (Spray 2021).

While unplanned, the combination of the two approaches-ethnographic visits and online interviews - strengthened the quality of data related to children's perspectives of asthma. Household visits involved a team of 2-3 researchers and allowed for multiple researcher perspectives of children's environments: their homes, neighborhoods, local resources, and family dynamics. These visits could be chaotic, with multiple children engaging in multiple activities simultaneously, and often with toddlers, guests, or unexpected changes of plans interrupting the research. Interviews could involve multiple contributors who moved in and out of the conversation, so that accounts of asthma management were co-constructed between caregivers and children. At other moments, different members of the research team talked to children or caregivers separately, though we generally followed the natural flux of interactions as we considered this likely reflected the relationship parents and children had in their asthma management also [Fig. 1].

The online interviews, by contrast, offered a quieter, more intimate window into children's experiences. One interviewer generally talked to one child alone. These interactions through Zoom elicited less contextual information about children's lives but facilitated more in-depth conversation and fostered relational processes as children could also see the interviewer in their space (Spray, Fechtel, and Hunleth in press). 
Table 3 Child and Household Research Activities

\begin{tabular}{|c|c|c|}
\hline Activity & $\begin{array}{l}\text { Household/online } \\
\text { project }\end{array}$ & Description \\
\hline $\begin{array}{l}\text { Asthma control } \\
\text { tests and } \\
\text { management } \\
\text { survey }\end{array}$ & Household and online & $\begin{array}{l}\text { Asthma Parent Proxy-Asthma control test } \\
\text { Child Asthma Control test } \\
\text { Asthma management survey (medication } \\
\text { use) }\end{array}$ \\
\hline Show and Tell & Household and online & $\begin{array}{l}\text { Ask children to show us and talk about (1) } \\
\text { their living space (2) Asthma medications } \\
\text { and paraphernalia }\end{array}$ \\
\hline Drawing & Household and online & $\begin{array}{l}\text { Invite children to draw a story about having } \\
\text { asthma and then use drawing as basis for } \\
\text { discussion }\end{array}$ \\
\hline Brainstorms & Household only & $\begin{array}{l}\text { For groups of children. Brainstorm topics } \\
\text { included: What does it mean to be a child/ } \\
\text { adult? What is asthma? }\end{array}$ \\
\hline $\begin{array}{l}\text { Semi-structured } \\
\text { interview (child } \\
\text { and/or sibling) }\end{array}$ & Household and online & $\begin{array}{l}\text { Questions about what asthma is, experiences } \\
\text { with asthma, experiences with health-care } \\
\text { providers, who is involved in asthma } \\
\text { management and what they do, children's } \\
\text { self-care and care for others }\end{array}$ \\
\hline $\begin{array}{l}\text { Child caregiving } \\
\text { survey }\end{array}$ & Online only & $\begin{array}{l}\text { Online survey about children's asthma care } \\
\text { activities }\end{array}$ \\
\hline $\begin{array}{l}\text { Welcome } \\
\text { pamphlet }\end{array}$ & Online only & $\begin{array}{l}\text { Invite children to write/draw/speak their } \\
\text { advice to a child who has just learnt they } \\
\text { have asthma }\end{array}$ \\
\hline Asthma stories & Online only & $\begin{array}{l}\text { Researcher and participant co-construct a } \\
\text { story about asthma. Researcher supplies } \\
\text { sentence beginnings and child fills in story }\end{array}$ \\
\hline
\end{tabular}

We also conducted 30-60 min phone or in-person interviews with 12 providers of asthma health care across the two sites. These providers, who we recruited through university medical networks, held a range of roles in asthma management, including pulmonologists, pediatricians, school nurses, nurse practitioners, a pharmacist and an asthma coach. Along with their perceptions of factors shaping family asthma management and access to care, we asked providers how they saw children's roles within asthma management and how they involved children in the clinical encounter.

The audio recordings were transcribed, and we collectively developed coding frameworks for each based on themes we identified in a sub-sample of transcripts and field notes. Through an iterative process of coding and data collection, and through summarizing individual cases and content with codes, we developed and refined the theory presented here. All participant names are pseudonyms (some participants chose their own). 


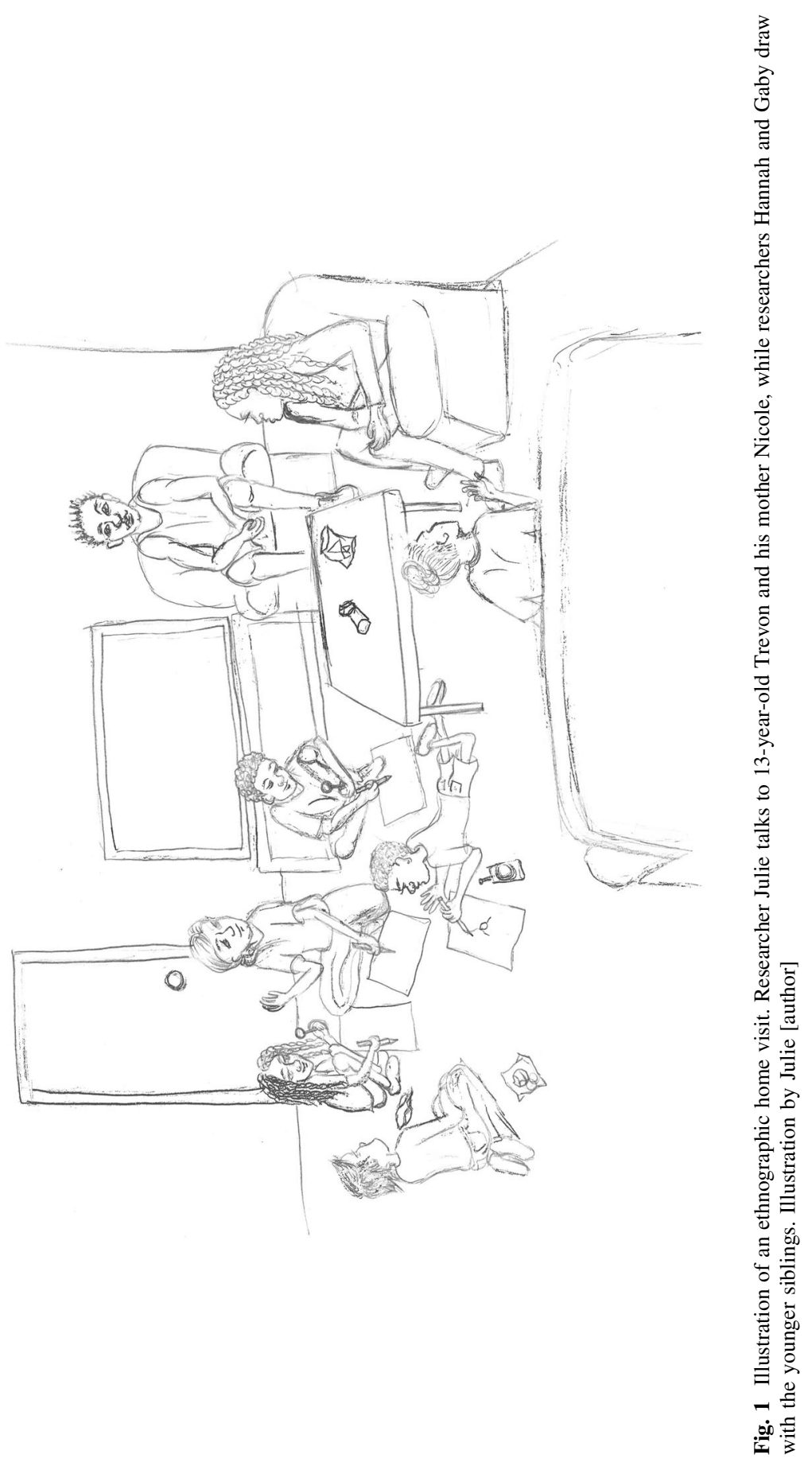




\section{Providers' Approaches to Children's Participation in Self-Management}

Before presenting children's perspectives, we first describe how the health-care providers we interviewed tended to view children's roles. Though children's invisibility in U.S. self-management policy and guidelines does not necessarily dictate how individual providers will view children's inclusion, the conceptual gap regarding children's participation has left asthma care providers to devise their own approaches to pediatric self-management. Evidence suggests in the U.S. a majority of children under 18 may not have received or do not recall asthma management education from a physician (Orrell-Valente et al. 2011). Indeed, few providers in our study mentioned how they involved or communicated with children until directly asked how they viewed children's roles. When prompted, few providers articulated an approach or philosophy of childhood, and they largely positioned children as passive recipients of care and education. Some discussed children's primary responsibility as learning to identify symptoms and figure out "what's going on in your body" (school health coordinator) and then to "tell an adult" (asthma coach). One pediatrician reported including children in discussions on asthma management but suggested their involvement was motivated by how much they wanted to "please the parent."

Providers held vague and idiosyncratic ideas about when and how children should become involved in their asthma care. Most providers tended to see adolescence as the period of transition in responsibility for asthma management, though there was no consensus on age, and "responsibility" usually meant remembering and self-administering medication. For example, when describing this process, one St. Louis pulmonologist centered pharmaceuticals within selfmanagement but indicated that transfer of responsibility was for the parent to "figure out":

"once the child becomes school-aged, um, there's something that that medication responsibility transfer should occur around eight- - t-t- - sorry, 10 years old. Um, I don't think that's great data on it, but that's some datathat's available. So, usually, by the time they turn 10, I start talking to the child — but I also look into the parent and say, "Hey, they-you gonna have to be the boss... um, and figure it out."

It is revealing to view providers' approaches within the sociology of childhood. Aside from the neoliberal emphasis on responsibility, child-centered scholars have noted that such constructions of children as apprentices for adulthood reflect Eurocentric, adult-centric biases grounded in patriarchal assumptions of the nuclear family_assumptions that often underpin child development research and pediatric medicine more broadly (Woodhead 2009). The primacy of developmental models has been challenged, particularly by education scholars, who demonstrate that rather than following a linear progression of cognitive 'stages', children's development is mediated by their experience; children can hold greater knowledge and skill in domains in which they have greater experience (Bluebond-Langner 1978; Yoos 1994). Further, in essentializing children as 'developing', developmental models 
turn childhood into a kind of holding space for adolescence, when they will transition into agentive people. This can erase the ways that both childhoods and adulthoods are interpolated with characteristics and practices associated with the other: how children can give care and adults be vulnerable; how children can be knowledgeable and adults naïve; and how neither are fully independent or dependent but both enmeshed within interdependencies of family and communities of care (Hunleth 2017, 2013).

The "apprenticeship" framing of asthma management therefore elides the way illness is situated within dynamic care relations and overlooks children as participants in their own care and as collaborators in family health. This also suggests that children's care needs may be inadequately met by health policy that focuses on caregivers while ignoring the processes through which children's asthma actually gets managed. What, then, might a perspective centering children reveal about how children acquire asthma management practices?

\section{The Child's Protocol}

We have previously described the "caregiver's protocol": the range of activities caregivers undertook (including environmental management, home remedies, avoiding activity, emotion management) that are primary to asthma management but generally absent from the clinical gaze (Spray et al. 2021). Caregiver's protocols, however, were a one-sided view of asthma management, and as an entry point to children's roles in asthma management, we found that collecting children's protocols revealed the breadth and sophistication of children's invisible self-care work. As with caregivers, we found that these "children's protocols" often resembled formalistic rules or procedures, though our term also encompasses reflexive or embodied practices that we identified through parsing children's stories or watching them demonstrate their bodily responses.

Ten-year-old Becca didn't use technical terms for her purple inhaler and yellow and blue spacers. But she knew how to use them; in the Zoom frame she slotted the inhaler into the spacer and showcased correct breathing technique with the deftness of a professional demonstrator. A patient of a severe asthma clinic in Gainesville, Florida, Becca had been well trained in the technical administration of her pharmaceuticals.

Becca's asthma management did not begin with inhaling pharmaceuticals, however-in large part because her inhaler was kept with the school nurse, not on her person. The advice she described to an imaginary child who has just learnt they have asthma involved a set of complex activities aimed at managing acute symptoms: sensing breathlessness, stopping, drinking water, calming, and getting help. Her asthma prevention work involved carrying water with her and avoiding a running program at school out of worry that the exercise would trigger an asthma attack. Finally, the asthma management she described was highly social—she emphasized the importance of telling people so they could get help, but she was also discerning about who to tell. Adults should know what to do, she told us, and other kids with asthma will know because they've experienced it, but sometimes kids can 
give you the wrong advice. Becca knew the three kids in her class with asthma, and she would support them to take deep breaths through their mouth, as her own "child's protocol" directed.

In highlighting children's self-care work here, we do not suggest that caregivers do not have key roles in children's asthma management practices. Rather children's protocols illustrated an asthma management that is not handed down unidirectionally from adult caregiver to child, but which children actively co-constructed. Perhaps as a result of their own experiences coping with acute asthma events, children's protocols tended to emphasize acute symptom management over the prevention strategies more common in caregiver's protocols. For example, Marie, a 16-year-old Black girl in Gainesville, detailed a step-by-step protocol that involved firstly drinking water (to see if "it'd go away"), then calming self-talk ("you really need to get air. Just sit down. Use your inhaler. It'll go away..."), getting an inhaler, and finally inhaling the medication while sitting back and breathing slowly. Marie's protocol contrasted with what we heard from her mother Monique, who described activities that included restricting Marie from exercise when she was younger, boiling water for steam, having her breathe peppers in a rag, avoiding marijuana and cleaning. Marie and her mother had therefore played different roles in Marie's asthma management, each generating sets of practices that attended to different demands of the disease.

If children acquire quite distinct self-management protocols, then the processes of their acquisition cannot resemble the unidirectional, linear handing over of responsibility as described in apprentice models. What, then, do the processes of developing self-management look like from children's perspectives? In the following sections, we unpack how children co-construct their asthma management with a range of other actors, through relational processes of care and responsibility, and within the spatial contexts and constraints of everyday childhoods.

\section{Co-constructing Asthma Management}

At age eight Trevon was playing baseball with older boys when he caught a fastpitch in the ribs. As he gasped for air, his 10-year-old brother Mike came, sat him down, told him to breathe. "The first person I saw, him helpin' me up," Trevon told Julie, while his mother Nichole listened.

The brothers competed though, until later that year when Mike collapsed at an after-school football team, and this time it was Trevon who helped his brother up and ran to the office to call their Mom.

That day the brothers had a conversation. "It wasn't emotional or anything," Trevon assured us. "We just talked about it together. I said, "We gotta stop this, 'cause, if me and him kept goin' back and forth, one of us is goin' to get hurt with asthma."

The boys made a new plan for how they would manage their asthma. They wouldn't push themselves so hard anymore, wouldn't compete. They would reduce the number of sports they played. They would encourage each other to ask for help. 
"We just start tellin' each other, and I'll be like, "Go get momma. You gotta make sure you're okay first." I'll stop [him] from layin' down. I'll be like, "What's wrong?" "I'm tired." "Go get your inhaler. Just go breathe a second so you aren't hurting yourself even worse.",

Rather than stoic silence, they attended to each other, learning the subtle signals of the other's distress: slowing, wheezing in the night. And they developed prevention strategies before football games, as Trevon described:

“Before we started, we'd just do breathin' together. We'll just breathe in and breathe out."

Trevon's story is striking for the way he locates key actors in his asthma management. He and his brother generated their asthma management protocols together based on their own goals: initially based in performative masculinity, physical prowess, and brotherly jostling for dominance; eventually based on mutual care and brotherly protection. His mother Nichole, who we had first interviewed for the caregiver project, was secondary to Trevon and his brother. Even Nichole placed herself outside the center of Trevon and Mike's asthma management. "No, I be on the sidelines," she interrupted when I asked Trevon how his mother was involved, meaning the literal sidelines of the football field, but also figuratively locating herself in relation to her sons' lives with asthma.

While the children we worked with weren't always the directors of their asthma care and caregivers weren't always sidelined, we saw children co-constructing their asthma management with a variety of actors-often excluding the main caregiver. This co-construction could involve siblings, like Trevon and Mike, friends, or in several cases, sports coaches. Following some incidents with kids having asthma attacks, Mike's school had instituted dedicated attention to breathing as part of football training. Students were required to take an online course and were coached in breathing technique, which Mike described as "deep tugs in through your nose, out through your mouth." Scott, a 13-year-old in St. Louis, also told us he had trained himself to control his asthma through breathing techniques he had developed with guidance from his boxing coach.

In our interviews with caregivers we had not heard any reports about the role sports coaches played in children's asthma management. This is the limitation of a caregiver-centric or dyadic views of asthma management; they overlook the other relationships through which children develop their asthma management. Asthma management is not a complete version handed from parent to child. Instead, having co-constructed an initial asthma management based on competition, Trevon and Mike continued to re-construct their strategies, first in collaboration with each other, then in partnership with coaches.

Although caregivers were not always involved, they were often present in children's asthma management protocols. Caregivers could often be children's partners in asthma management; for example, when Marie had an asthma exacerbation at home, her protocol included her mother fetching her inhaler, water, and a cool rag to put on Marie's forehead. While Marie and her mother here cooperatively co-created this protocol, other times, however, the co-construction 
process might involve children resisting or avoiding their caregiver's intervention, as did Trevon and Mike when they concealed their symptoms from Nichole. Coconstruction, therefore, could involve co-constructing against or around as well as co-constructing with. Yet in overlooking children's roles in their health, a pediatric asthma management policy based on ideals of individualism and parental responsibility places the burden and blame for asthma exacerbations on Nichole, who as a parent is expected to be an all knowing and all powerful manager of passive and dependent children. In her original caregiver interview, we had heard Nichole return repeatedly to blame herself for not being able to realize when her sons were struggling to breathe. Doctors had told her how to identify symptoms, "but I'm just am-I have-I have failed to learnin' about it," she sighed. "I waited so long, like I just did not call it good." It was only talking to Trevon that we all heard that the boys' asthma management was not as much in her hands as she-and possibly her doctors-had thought.

\section{Managing Asthma Within the Responsibilities of Care}

Clinical perspectives generally view care as flowing unidirectionally from parent to child, care giver to care receiver, or in the form of self-care: individuals "taking responsibility" for following medical recommendations to ensure their own health maintenance. Social scientists, of course, know that care is much more complex and dynamic than this view. For children, care is deeply implicated in both their identities as children and of their very survival (Hunleth 2017). Though the structures of societies often render them vulnerable or dependent, children are not passive dependents in their family relations, but actively co-construct interdependencies. Children's social positions in the family or with friends is established, in large part, through enacting care, whether this be material contributions to household finances or labor, care for health, affective or imaginal care (Hunleth 2019; Luttrell 2020; Spray 2020). Through their care for themselves or others, children can strengthen relationships, support household functioning, and secure their social survival.

The asthma management practices children co-construct are therefore embedded within these broader interdependencies and dynamics of care. For children whose survival is already threatened or insecure, for example, due to social marginalization, material deprivation, or family instability, these care dynamics may be particularly sharp. In our study, children like Lion Tamer, who lived with his single mother in housing subsidized for individuals with disabilities, were deeply enmeshed in reciprocities of care that shaped their asthma management.

Lion Tamer, an African American ten-year-old with big dimples and short braided hair who chose his own pseudonym, did not say much for the first half-hour of our visit to the small unit that he shared with his mother Pamela in an impoverished neighborhood in Gainesville. Instead the three researchers who visited heard Pamela's stories: about the circumstances of Lion Tamer's birth; the bus accident six months earlier that injured them both; the traumatic days after Lion 
Tamer had a brain shunt malfunction. Lion Tamer sat quietly, staying close to his mother, smiling and nodding.

After one of our team drew Pamela into conversation in the other side of the room, we heard Lion Tamer's perspectives. "It sounds like your mom takes care of you a lot," another researcher, Hannah, reflected, "but do you also take care of yourself?".

"I take care of myself and my mom," Lion Tamer replied. "If my mom is sick in bed, I make breakfast or at least find something to make for my mom, and then I bring it to her. Then, I make breakfast for me. Then, if she feels better, she can walk me to school. If she doesn't feel better, then I just stay home caring for her."

"Does it ever get in the way of what you wanna do?" Hannah, asked.

"If you mean by sleep and my mom wakes me up, then yes," Lion Tamer answered. His mother would sometimes enter his room to check his breathing while he slept. Then looking across the room, Lion Tamer added, "I thought I heard my mom crying?"

"I think she got very passionate. She cares about you a lot," the third researcher, Julie, answered, and Gaby, the team member talking with Pamela, reassured him, "She was tellin' us how much she loves you, Lion Tamer."

Although we had come to visit Lion Tamer about his asthma, he and Pamela were not co-constructing his asthma management in a vacuum. This was an asthma management woven into lives where a mother's advocacy for her child's brain shunt replacement was reciprocated by a child's concern for his mother's tears, and, after his mother watched him breathe at night, a son missed school to tend to his mother's illness in the morning. These interdependent, affective relations of mutual care that scaffold asthma management are in turn shaped by the socio-economic and racialized realities of domestic life.

Children could care for themselves in order to care for parents, or avoid seeking care in order to protect parents. For Trevon and Mike, introduced in the previous section, taking care of themselves was a way that they could care for their mother. As they explained, "We can't just think that, every time we need help, she's gonna be able to come to our rescue when we're adults because she has to be an adult too. She has to work." Their younger sister, six-year-old Kennedy, experienced asthma as a sore neck, but teared up as she added "I'm scared to tell my mom." She couldn't say why she was scared, but Nichole had told us of her own distress when Kennedy had a dramatic exacerbation after running around at her brothers' football game. "I-I don't do good in medical emergencies," Nichole had said, returning, again, to blame herself for an asthma management she had never been trained to deal with.

While Trevon, Mike, and Kennedy protected their mother from distress in the ways they best knew, children could attend to other family needs. Jenny, a nurse at a St. Louis elementary school with a large proportion of low-income students, described how children would sometimes arrive at the school clinic in a late stage of asthma exacerbation, having attempted to conceal their symptoms earlier in the day. 
Of a 12-year-old whom she sent to the emergency room one day, she explained: "Both parents work nights. This student never came to see me. [...He was thinking] I don't wanna interfere with my parents' work schedule." In these cases, children were "taking responsibility" for "self-managing their symptoms," but not in the way Jenny, or other providers, would like. A context where caregiving leave is usually unavailable to low-income employees creates competing responsibilities (Trnka and Trundle 2017) for children who are also aware of the need to protect their family's income.

Children's caregiving in these circumstances might be considered "parentification," or as an inappropriate burdening of children with responsibilities that should belong to adults in accordance with the view that children ought not be responsible. Such views can be Eurocentric and classed, overlooking the way that marginalized families, particularly in African American communities, rely on reciprocal domestic networks to provide critical resources to buffer against scarcity (Stack 1974). We observed children's care for others many times throughout our home visits: boys like Scott and Trevon would rise during interviews to pick up babies, open snack packets, translate between us and a younger sibling, or intercede when younger siblings were crying or fighting. While 8-year-old Josiah picked up and soothed his baby sister, his family's retelling of the story of his recent asthma attack focused on how, when Josiah was too sick to get up from bed, his 3-year-old brother had dragged him into the hallway and alerted their mother, and of how the younger siblings climbed into the hospital bed with Josiah and gave him their toys. Having co-constructed their own asthma management as children, Trevon and Mike spoke confidently of how they now cared for their younger brother and sister's asthma, watching them when they played sport and helping them to identify when they needed to slow down. Care and responsibility are the raw materials through which children co-construct the interdependencies vital for their security and flourishing. But these interdependencies can sit at odds with a health agenda that assumes children to be either passive recipients of parental care, or as transitioning toward a developmental end-point of independent self-care. The interdependencies through which asthma management is co-constructed involve "taking" different kinds of responsibilities, and emphasize care with and for others over self-care. By creating opportunities for children to make contributions of value to their family or community, such interdependencies also make children valuable and valued.

Beyond children's care for family, children also co-construct interdependent care relationships with friends who provide critical social support with navigating the stresses of authority structures, social hierarchies, and even physical threats at school or home (Spray 2020; Spray et al. 2018). While other studies have reported that asthma can be stigmatized among school-age children (Monaghan and Gabe 2018), Sallad, a 13-year-old white boy in St. Louis, told us that when he uses his inhaler, his friends “know I'm havin' a little bit of trouble breathin'. They'll be like, "You good, bro?", This care for asthma fit into a wider friendship culture where "we all look out for each other." Similarly, Becca (10) described how her friend helped another child with asthma symptoms the day before: "she would say "stop," get you to calm down, tryin' to get you to take deep breaths in and out and stuff and get you to a teacher." 
These relationships were not only important to asthma management, but in some cases asthma management was important to creating relationships. We saw such dynamics in the way that five-year-old Mason would, according to his mother, simulate asthma symptoms in order to elicit her care, and in how Lion Tamer would tolerate his mother waking him in the night to administer medication he felt he didn't need. Children's self-management may involve management-by-others, not because they are incapable of "taking responsibility," but because care is a way in which parent-child relationalities are enacted (Spray 2020), and allowing parents to give care is important to maintaining relationships of love, safety, and protection. For a child to care for themselves implies a child who does not have someone who cares for them.

\section{Generating Practices Within the Structural Constraints of Childhoods}

We have previously reported how systemic forces such as racial and socioeconomic disadvantage and the privatization of medicine shaped the nature of caregiver's protocols (Spray et al. 2021). Where access to health care, or health care itself is unreliable and resources constrained, families construct management regimes that utilized the resources they could access more readily. Structural constraints also shaped children's protocols, but these included the specific constraints of childhood that dictate what children can and cannot control: school policy and institutional authority, medical gatekeepers, mobility.

These structural constraints of childhood also intersect in particular ways with a biomedical approach to self-management that centers pharmaceuticals. A rescue inhaler can be powerfully effective at restoring breath, but children may not have access to an inhaler at a time of need, including because of institutional policies that disallowed children from carrying their inhalers with them and required medication to be held by a school nurse. This was the case for Becca (10), who lamented, "I'm sayin' I wanna know, what if I'm at tutorin'? Because my tutorin' is at school campus, and the nurse is not there. What if I have a asthma attack there? How am I gonna use it and the nurse is not there to help me?" Similarly, when the researcher asked six-year-old Unicorn if she had a nurse at school who helped her, Unicorn responded, "they leave at the end of the day. There's nobody goin' to nurse." Unicorn's emphasis on the time when the nurse wasn't there indicated her acute awareness of how her access to her inhaler was institutionally mediated. Children's asthma could be triggered in the playing that occurred after school, making this a period vulnerable to asthma risk. The geographic distance between themselves and their inhaler also created stress for children because a state of breathlessness precluded them from walking across the school from the playground or gym where they had experienced the onset of asthma symptoms to the teacher, nurse, or locker room where their inhaler was kept. This inability to carry one's own inhaler affected younger children more, as older children would more commonly be permitted to carry medication with a doctor's note. Some children who were not permitted to carry an inhaler did so anyway; 13-year-old Sallad explained that rather than make a special appointment each year to obtain a doctor's note for permission to carry his 


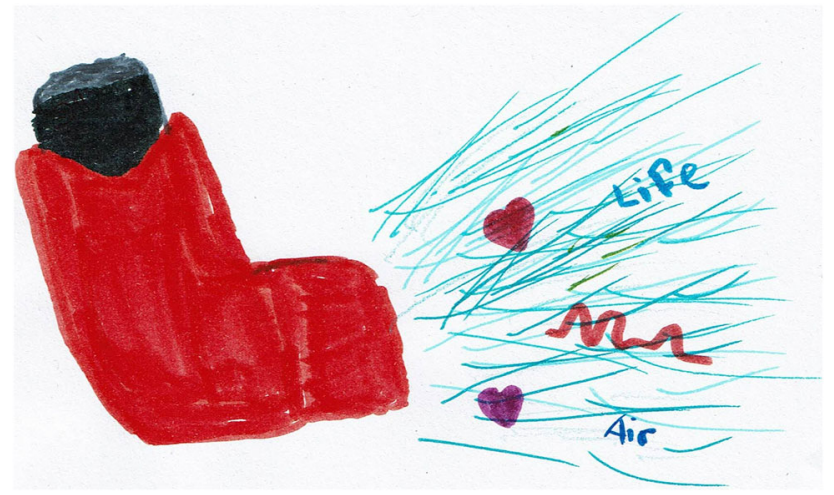

Fig. 2 Drawing by Courtney, age 12

inhaler, he would hide his inhaler in his pocket and "I'd go to my locker and just open it, act like I'm grabbin' somethin', and take a puff." Even when children had permission to carry an inhaler, this came with the mental labor of keeping access to the inhaler that could be easily lost, forgotten, misplaced, emptied, or expire.

The prominence of inhalers in children's drawings reflected their importance to children; 12-year-old Courtney's drawing of what it's like to have asthma depicted an inhaler spraying heart and heartbeat symbols with the words "life" and "air" because, as Courtney explained, "if you didn't have [the inhaler], you'd probably lose your life because, if you can't breathe and calm down, then your heart would stop breathing" [Fig. 2]. Other children stressed the importance of access to inhalers. In her 'pamphlet' 15-year-old Esther made three separate references to the importance of keeping an inhaler nearby; she keeps inhalers in multiple locations including the bathroom, her mom's purse, and her friend's car. For 13-year-old Scott, remembering to carry his inhaler had been a challenge and he had cultivated a habit of permanently keeping his inhaler in his coat pocket and transferring the inhaler to other garments when weather necessitated. Experiencing the negative consequences of not having an inhaler when needed could precipitate a habitual, embodied vigilance toward inhaler accessibility.

On the other hand, if inhalers are not always accessible, children needed to develop alternative strategies for asthma management. For children like Becca, the role of friends was important in part because friends could fetch an adult to access their inhaler. Further, a strikingly ubiquitous feature of children's narratives was the use of water. Children used water for diagnostic purposes, as an initial intervention, or to augment or replace treatment with albuterol. For example, when asked as part of an ACT what medications he had taken for asthma in the last month, Lion Tamer replied: "Proair [albuterol], and the second one is water." When we interviewed 10-year-old Jasmine she did not have any asthma medication in the house because her inhalers had run out three months ago and her nebulizer had broken. Instead, many of the asthma management strategies she described involved drinking water. 
When she awoke in the night coughing from the dusty old mattress she slept on with her sister, she told us, "I be wakin' up 3:00 a.m. in the morning, gettin' a water, drinkin' it, and then go back to sleep." When she got breathless during the day, she would "sit outside and drink water." Courtney told us she drank water after taking her inhaler when her asthma was triggered by pet fur as it could take a few minutes for the symptoms to dissipate. Rick (13) took four water bottles with him on a plane to help manage his anxiety, which he considered a cause of asthma attacks. Marie used water for acute symptoms and also for health maintenance as part of overall asthma prevention, as she describes:

“it does help. It really does help asthma. I don't know. Just if you do drink more water-I don't know. I'm not saying it'll help prevent it, but it willwhen your body needs that extra umph, it'll have it."

While some caregivers also emphasized water-notably, Marie's mother Monique-overall water featured far less in caregiver narratives except when caregivers were describing their child's practices: One caregiver told us of her daughter: "She drink a lotta water. I asked, "What do the water do?" She told me, "Mama, I don't know. Mama, let me get some water." Another caregiver described finding her child coughing from dog hair when she picked him up at a neighbor's house. "I was like, "Honey, why didn't you tell her?" "Well, I just-I-I-I asked for water." And she said, "Oh, yeah. He kept askin' for water." I'm like, ”He doesn't—he doesn't need water. He needs his inhaler,"” she recounted.

Water may have some therapeutic benefits, but for children the advantage over inhalers lies in water's accessibility. Water fountains are present and close for children in a gym class or on the playground. Friends' homes have water. Drinking water may also be more socially acceptable. Children's self-management practices, therefore, are not only co-constructed with caregivers to include strategies for times of scarcity, but also in response to the specific constraints and available resources of childhood.

\section{Discussion}

The problem with organizing children's asthma management around cultural conceptions of children as people who ought not hold critical roles in their health care, is that children $d o$ hold critical roles. By centering children in our investigation of asthma management, we heard how they generate their own protocols for selfmanagement and how they make decisions about treatment or whether to seek or accept help. These practices, many of which were unknown to or unrecognized by caregivers, fell well outside of the apprentice model that many providers implicitly worked from. Moreover, children's self-management protocols began well before pharmaceuticals, the centerpiece of clinical management, entered the picture, in large part because children's access to inhalers was so mediated. This, we argue, demonstrates the importance of interpreting what children do in the contexts of childhood's particular structural and relational positionings. 
In arguing such, we build upon a foundation of literature in the social science of child health that has established that children are actors who employ agency in managing their health (Bluebond-Langner 1978; Clark 2003; Christensen 1999; Hunleth 2017; Mayall 1993; Prout 1986) and do so within the range of options available to them as constrained by the structures of society, access to power and resources, and the agency of others (Bluebond-Langner and Korbin 2007; Allerton 2016; Hunleth 2019; Spray 2020). This view of children as social actors remains unfortunately niche in contrast to the dominant cultural constructions of children as incompetent and passive recipients of care that persistently underpin pediatric medicine. As we continue to advocate for medical fields and health policy to recognize and take seriously children's agency, we also refine our childhood studies lenses for understanding children's roles in their health care. Agency and constraint are crucial but crude building blocks for understanding why children do what they do-and how we can best support them.

By conceptualizing children as co-constructors, we shift focus from the child as an individual enacting agency in response to constraint, and emphasize the dispersed nature of the relationships through which children co-produce asthma management-and the competing priorities that accompany those relationships: identities, obligations, interdependencies. Through such a relational lens, we can see a child's request for water instead of an inhaler not as ignorance but as inventive in contexts of lives governed by powerful adults and structures, and concealing symptoms from adults not as incompetence but as a child's awareness of their competing vulnerabilities and responsibilities generated from childhood's social precarity. While adults can dismiss children's activities as inconsequential (Spray 2020), these practices matter because they directly shape children's health status and vulnerability to life-threatening exacerbations, and they mediate children's experiences of childhood-whether the trauma of ED visits or the relationshipbuilding care they give and receive.

Including children without overburdening them with tasks and responsibilities that are beyond their skills is a delicate balance (this issue also applies to adults (Anderson et al. 2016; Spray et al. 2021)). We certainly do not advocate that the neoliberal responsibilization of adult citizens be extended to children. Rather, we argue that a health policy based on individual self-management must recognize that children are inevitably implicated in this care work long before adolescence. Substituting child patients with adult caregivers obscures the uncomfortable realities that children have agency and that adults cannot know or control all aspects of their health. Leaving children as shadows in policy or guidelines also means leaving unaccounted for the other actors, interdependencies, and constraints that substantively shape children's health practices (Hunleth 2017). Ignoring these realities does children a disservice, not only by denying their rights to participation, but also by under-equipping children to carry out tasks necessary to their health. Equating parent-proxies with patients also places unrealistic expectations on parents, who are held responsible - and blamedfor a health status they have even less influence over than do patients themselves.

Some providers we spoke to, particularly pediatricians, school nurses, and community health workers, made efforts to include children in explanations of asthma, medications, and inhaler technique. However, children's inclusion must go 
beyond seeing them as recipients of education (Freire 1973; hooks 2003). Asthma self-management involves a great deal more than receiving pharmaceuticals-it necessitates a complex set of activities including bodily and environmental awareness and monitoring, memory work, emotion management, communication, and planning and organization that even adult patients struggle with (Apps et al. 2019). How these activities are recognized and organized between children and other family members is foundational to successful asthma management. Yet these activities are largely absent from asthma guidelines focused on pharmaceuticals, and providers are time-constrained and poorly equipped to facilitate conversations that would guide families with identifying and negotiating children's roles and support. A systemic overhaul of pediatric health-care training and delivery using a child-centered lens is needed (Ford et al. 2018), along with a model for children's participation in chronic asthma management that goes beyond age standardized developmental expectations and shifting responsibility onto parents. Asthma education should not be treated as a set of lessons to be internalized within a specific developmental period but as a space for children to work out their selfdetermination.

Acknowledgements We are supported in this research by principal investigators Erika A. Waters and James Shepperd. Hannah Fechtel and Sienna Ruiz undertook significant field and online research activities, with additional support from Gaby Pogge, Julia Maki, and Cassidy Sykes. Jean Hunleth and Julia Maki conducted St. Louis caregiver interviews and David Fedele and Rachel Forsyth conducted Florida caregiver interviews. Sreekala Prabhakaran and David Fedele assisted with recruitment and advised from clinical perspectives. This study was supported by funding from the National Heart, Lung, and Blood Institute (NHLBI) of the National Institutes of Health (NIH), R01HL137680 (MPI: Shepperd and Waters).

Funding This study was funded by the National Heart, Lung, and Blood Institute (NHLBI) of the National Institutes of Health (NIH), R01HL137680 (MPI: Shepperd and Waters).

\section{Declarations}

Conflict of interest On behalf of all authors, the corresponding author states that there is no conflict of interest.

Ethical approval All procedures performed in studies involving human participants were in accordance with the ethical standards of the institutional and/or national research committee and with the 1964 Helsinki declaration and its later amendments or comparable ethical standards.

Informed consent Informed consent was obtained from all individual participants included in the study.

\section{References}

Allerton, Catherine

2016 Introduction: Encountering Children. In Children: Ethnographic Encounters, pp. 1-15. New York: Bloomsbury. 
Apps, Lindsay D, Stacey Chantrell, Sally Majd, Elizabeth Eglinton, Sally J Singh, Anna C Murphy, Peter Bradding, Ruth H Green, Nicky Hudson, and Rachael A Evans

2019 Patient Perceptions of Living with Severe Asthma: Challenges to Effective Management. The Journal of Allergy and Clinical Immunology In Practice 7(8):2613-2621.e1. https://doi.org/ 10.1016/j.jaip.2019.04.026.

Aroni, R, Susan Sawyer, Michael Abramson, Kay Stewart, Frank Thien, Dianne Goeman, and Jo Douglass

2003 Asthma Self-Management: What Do We Really Mean?. Australian Journal of Primary Health 9:10-17.

Bluebond-Langner, Myra

1978 The Private Worlds of Dying Children. Princeton, NJ: Princeton University Press.

Bluebond-Langner, Myra, and Jill E Korbin

2007 Challenges and Opportunities in the Anthropology of Childhoods: An Introduction to 'Children, Childhoods, and Childhood Studies'. American Anthropologist 109(2):241-46.

Brown, Nicola, Robyn Gallagher, Cathrine Fowler, and Sandra Wales

2010 The Role of Parents in Managing Asthma in Middle Childhood: An Important Consideration in Chronic Care. Collegian 17(2):71-76.

Buford, Terry A

2004 Transfer of Asthma Management Responsibility from Parents to Their School-Age Children. Journal of Pediatric Nursing 19(1):3-12.

Christensen, Pia Haudrup, and Allison James

2000 Research with Children: Perspectives and Practices. London/New York: Falmer Press.

Christensen, Pia Haudrup.

1999. Towards an Anthropology of Childhood Sickness: An Ethnographic Study of Danish Schoolchildren. Doctoral Thesis, University of Hull.

City of St. Louis Department of Health.

2018. Asthma Data Brief. St. Louis: Center for Health Information, Planning, and Research. https:// www.stlouis-mo.gov/government/departments/health/documents/upload/Asthma-Data-Brief-0320-2018-2-hb.pdf.

Clark, Cindy Dell

2003 In Sickness and in Play: Children Coping with Chronic Illness. New Brunswick, NJ: Rutgers University Press.

Clark, A, and P Moss

2011 Listening to Young Children: The Mosaic Approach. 2nd Edition. London: National Children's Bureau for the Joseph Rowntree Foundation.

Creer, Thomas L

1976 Chronically Ill and Handicapped Children, Their Management and Rehabilitation. Champaign, Ill.: Research Press Co.

Davis, KJ, R Disantostefano, and DB Peden

2011 Is Johnny Wheezing? Parent-Child Agreement in the Childhood Asthma in America Survey. Pediatric Allergy and Immunology 22(1-Part-I):31-35.

duRivage, Nathalie, Michelle Ross, Stephanie L Mayne, Andrew Suh, Daniel Weng, Robert W Grundmeier, and Alexander G Fiks

2017 Asthma Control Test: Comparing Parent Proxy with Parent and Child Report for Children 6 to 12 Years. Clinical Pediatrics 56(4):341-47.

Ekim, Ayfer, and Ayse Ferda Ocakci

2013 Perceptions of Parents and Children Regarding Asthma Management Responsibilities. Journal for Specialists in Pediatric Nursing 18(4):289-96. https://doi.org/10.1111/jspn.12037.

Florida Department of Health.

n.d. Florida Health Charts. http://www.flhealthcharts.com/charts/ChronicDiseases/default.aspx. Accessed 12 Feb 2020.

Ford, Karen, Annette Dickinson, Tineke Water, Steven Campbell, Lucy Bray, and Bernie Carter

2018 Child Centred Care: Challenging Assumptions and Repositioning Children and Young People. Journal of Pediatric Nursing 43(November):e39-43. https://doi.org/10.1016/j.pedn.2018.08.012.

Freire, Paulo.

1973. Education for Critical Consciousness. [1st American ed.]. New York: Continuum. 
Gabe, Jonathan, Gillian Olumide, and Michael Bury

2004 'It Takes Three to Tango': A Framework for Understanding Patient Partnership in Paediatric Clinics. Social Science \& Medicine 59(5):1071-79.

García-Sánchez, Inmaculada M

2018 Children as Interactional Brokers of Care. Annual Review of Anthropology 47(1):167-84. https://doi.org/10.1146/annurev-anthro-102317-050050.

Global Initiative for Asthma (GINA).

2010. "Global Strategy for Asthma Management and Prevention." Global Initiative for Asthma. http:// www.ginasthma.org/.

Guggenheim, Martin

2005 What's Wrong with Children's Rights. Cambridge, MA: Harvard University Press.

Guyatt, Gordon H, Elizabeth F Juniper, Lauren E Griffith, David H Feeny, and Penelope J Ferrie

1997 Children and Adult Perceptions of Childhood Asthma. Pediatrics 99(2):165-68.

Harris, Kelly M

2019 Mapping Inequality: Childhood Asthma and Environmental Injustice, a Case Study of St. Louis, Missouri. Social Science \& Medicine 230(June):91-110. https://doi.org/10.1016/ j.socscimed.2019.03.040.

hooks, bell.

2003. Teaching Community: A Pedagogy of Hope. London, UNITED KINGDOM: Taylor \& Francis Group. http://ebookcentral.proquest.com/lib/auckland/detail.action?docID=1192710.

Hunleth, Jean

2011 Beyond on or with: Questioning Power Dynamics and Knowledge Production in 'ChildOriented' Research Methodology. Childhood 18(1):81-93. https://doi.org/10.1177/ 0907568210371234.

2013 'ARVs' as Sickness and Medicine: Examining Children's Knowledge and Experience in the HIV Era in Urban Zambia. AIDS Care 25(6):763-66.

2017 Children As Caregivers: The Global Fight Against Tuberculosis and HIV in Zambia. New Brunswick, NJ: Rutgers University Press.

2019 Zambian Children's Imaginal Caring: On Fantasy, Play, and Anticipation in an Epidemic. Cultural Anthropology 34(2):155-86. https://doi.org/10.14506/ca34.2.01.

Hunleth, Jean, Julie Spray, Sienna Ruiz, Julia Maki, David Fedele, Sreekala Prabhakaran, Rachel Forsyth, et al.

2020 Situating Household Management of Children's Asthma in the Context of Social, Economic, and Environmental Injustice. Journal of Asthma. https://doi.org/10.1080/02770903.2020.1837159.

James, Allison

1993 Childhood Identities: Self and Social Relationships in the Experience of the Child. Edinburgh: Edinburgh University Press.

2013. Socialising Children. Studies in Childhood and Youth. Basingstoke: Palgrave Macmillan.

James, Allison, Chris Jenks, and Alan Prout

1998 Theorizing Childhood. Cambridge: Polity Press.

Kieckhefer, Gail M, and Cristine M Trahms

2000 Supporting Development of Children with Chronic Conditions: From Compliance toward Shared Management. Pediatric Nursing 26(4):354.

Kieckhefer, Gail M., Cristine M. Trahms, Shervin Churchill, and Jessica N. Simpson.

2009. Measuring Parent-Child Shared Management of Chronic Illness. Pediatric Nursing 35 (2).

Laurel, Anderson, Jelena Spanjol, Josephine Go Jefferies, Amy L. Ostrom and Courtney Nations

BakerSterling A. Bone, Hilary Downey, Martin Mende, and Justin M. Rapp

2016 Responsibility and Well-Being: Resource Integration under Responsibilization in Expert Services. Journal of Public Policy \& Marketing 35(2):262-79. https://doi.org/10.1509/ jppm.15.140. 
Liu, Andrew H, Robert Zeiger, Christine Sorkness, Todd Mahr, Nancy Ostrom, Somali Burgess, Jacqueline Carranza Rosenzweig, and Ranjani Manjunath

2007 Development and Cross-Sectional Validation of the Childhood Asthma Control Test. Journal of Allergy and Clinical Immunology 119(4):817-25.

Luttrell, Wendy

2020 Children Framing Childhoods: Working-Class Kids' Visions of Care. 1st Edition. Great Britain: Policy Press.

Mammen, Jennifer, Hyekyun Rhee, Sally A Norton, Arlene M Butz, Jill S Halterman, and Kimberly Arcoleo

2018 An Integrated Operational Definition and Conceptual Model of Asthma Self-Management in Teens. Journal of Asthma 55(12):1315-27. https://doi.org/10.1080/02770903.2017.1418888.

Mayall, Berry

1993 Keeping Healthy at Home and School: 'it's My Body, so It's My Job ‘. Sociology of Health \& Illness 15(4):464-87.

McQuaid, Elizabeth L, Sheryl J Kopel, Robert B Klein, and Gregory K Fritz

2003 Medication Adherence in Pediatric Asthma: Reasoning, Responsibility, and Behavior. Journal of Pediatric Psychology 28(5):323-33.

Meah, Angela, Peter Callery, Linda Milnes, and Sam Rogers

2010 Thinking 'Taller': Sharing Responsibility in the Everyday Lives of Children with Asthma. Journal of Clinical Nursing 19(13-14):1952-59.

Modi, Avani C, Ahna L Pai, Kevin A Hommel, Korey K Hood, Sandra Cortina, Marisa E Hilliard, Shanna M Guilfoyle, Wendy N Gray, and Dennis Drotar

2012 Pediatric Self-Management: A Framework for Research, Practice, and Policy. Pediatrics 129(2):e473-85.

Monaghan, Lee F., and Jonathan Gabe.

2018. Managing Stigma: Young People, Asthma, and the Politics of Chronic Illness. Qualitative Health Research, 1049732318808521.

Munzenberger, Paul, Elizabeth Secord, and Ron Thomas

2010 Relationship between Patient, Caregiver, and Asthma Characteristics, Responsibility for Management, and Indicators of Asthma Control within an Urban Clinic. Journal of Asthma 47(1):41-45.

Newbould, Jennifer, Felicity Smith, and Sally-Anne Francis

2008 'I'm Fine Doing It on My Own': Partnerships between Young People and Their Parents in the Management of Medication for Asthma and Diabetes. Journal of Child Health Care 12(2):11628.

NHLBI

2007 Expert Panel Report 3 (EPR-3): Guidelines for the Diagnosis and Management of AsthmaSummary Report 2007. The Journal of Allergy and Clinical Immunology 120(5 Suppl):S94.

2020 Focused Updates to The Asthma Management Guidelines: A Report from the National Asthma Education and Prevention Program Coordinating Committee Expert Panel Working Group. Bethesda, MD: National Health Lung and Blood Institute. https://www.nhlbi.nih.gov/sites/default/ files/publications/AsthmaManagementGuidelinesReport-2-4-21.pdf.

Nieuwenhuys, O

1996 The Paradox of Child Labor and Anthropology. Annual Review of Anthropology 25:237-51.

Orrell-Valente, Joan K, Leah G Jarlsberg, Laura G Hill, and Michael D Cabana

2008 At What Age Do Children Start Taking Daily Asthma Medicines on Their Own?. Pediatrics 122(6):e1186-92. https://doi.org/10.1542/peds.2008-0292.

Orrell-Valente, Joan K, Kimberley Jones, Stephanie Manasse, Shannon M Thyne, BuddN Shenkin, and Michael D Cabana

2011 Children's and Parents' Report of Asthma Education Received from Physicians. Journal of Asthma 48(8):831-38. https://doi.org/10.3109/02770903.2011.604882.

Petsios, Konstantinos, Kostas N Priftis, Constantinos Tsoumakas, Elpis Hatziagorou, John N Tsanakas, Petros Galanis, George Antonogeorgos, and Vasiliki Matziou

2011 Level of Parent-Asthmatic Child Agreement on Health-Related Quality of Life. Journal of Asthma 48(3):286-97. https://doi.org/10.3109/02770903.2011.555031. 
Pradel, Francoise G, Abraham G Hartzema, and Patricia J Bush

2001 Asthma Self-Management: The Perspective of Children. Patient Education and Counseling 45(3):199-209.

Prout, Alan

1986 'Wet Children'and 'little Actresses': Going Sick in Primary School. Sociology of Health \& Illness 8(2):113-36.

Prout, Alan, L Hayes, and L Gelder

1999 Medicines and the Maintenance of Ordinariness in the Household Management of Childhood Asthma. Sociology of Health \& Illness 21:137-62.

Reynolds, Pamela.

1991. Dance, Civet Cat: Child Labour in the Zambezi Valley. Baobab Books; London: Athens: Zed Books; Ohio University Press.

Spray, Julie

2020 The Children in Child Health: Negotiating Young Lives and Health in New Zealand. New Brunswick, N.J.: Rutgers University Press. https://doi.org/10.2307/j.ctvvh85fc.

2021 Drawing Perspectives Together: What Happens When Researchers Draw with Children?. Visual Anthropology Review. https://doi.org/10.1111/var.12244.

Spray, Julie, Bruce Floyd, Judith Littleton, Susanna Trnka, and Siobhan Mattison

2018 Social Group Dynamics Predict Stress Variability among Children in a New Zealand Classroom. HOMO 69(1-2):50-61.

Spray, Julie, Chelsey Carter, Erika A Waters, and Jean Hunleth

2021 Not Breathing Easy: 'Disarticulated Homework' in Asthma Management. Medical Anthropology Quarterly 35(2):285-302.

Spray, Julie, Hannah Fechtel, and Jean Hunleth.

In Press. What Do Arts-Based Methods Do? A Story of (What Is) Art and Online Research with Children during a Pandemic. Sociological Research Online.

Stack, Carol

1974 All Our Kin. New York, NY: Basic Books.

Sweis, Rania Kassab

2021 Paradoxes of Care: Children and Global Medical Aid in Egypt. Stanford, CA: Stanford University Pres.

Trnka, Susanna

2017 One Blue Child: Asthma, Personal Responsibility, and the Politics of 21st Century Patienthood. Stanford, CA: Stanford University Pres.

Trnka, Susanna, and Catherine Trundle.

2017 Competing Responsibilities: The Ethics and Politics of Contemporary Life. Duke University Press.

Wade, Shari, Shaheen Islam, Gary Holden, Deanna Kruszon-Moran, and Herman Mitchell

1999 Division of Responsibility for Asthma Management Tasks Between Caregivers and Children in the Inner City. Journal of Developmental \& Behavioral Pediatrics 20(2):93-98.

Walders, Natalie, Dennis Drotar, and Carolyn Kercsmar

2000 The Allocation of Family Responsibility for Asthma Management Tasks in African-American Adolescents. Journal of Asthma 37(1):89-99.

Winkelstein, Marilyn L, Karen Huss, Arlene Butz, Peyton Eggleston, Perla Vargas, and Cynthia Rand

2000 Factors Associated with Medication Self Administration in Children with Asthma. Clinical Pediatrics 39(6):337-45.

Woodhead, M

2009 Child Development and the Development of Childhood. In Handbook of Childhood Studies. Jens Qvortrup, William A Corsaro, and Michael-Sebastian Honig, eds., pp. 46-61. London: Palgrave.

Yoos, H. Lorrie

1994 Children's Illness Concepts: Old and New Paradigms. Pediatric Nursing 20(2):134.

Publisher's Note Springer Nature remains neutral with regard to jurisdictional claims in published maps and institutional affiliations. 